Bundesgesundheitsbl -

Gesundheitsforsch - Gesundheitsschutz

2000 • 43:323-327 @ Springer-Verlag 2000
Leitthema: Umwelt und Gesundheit

B. Seifert ${ }^{1} \cdot$ H. Schreiber ${ }^{1} \cdot$ B. Bellach ${ }^{2} \cdot$ U. Gundert-Remy ${ }^{3} \cdot$ T. Jung $^{4}$

${ }^{1}$ Umweltbundesamt, Berlin

${ }^{2}$ Robert Koch-Institut, Berlin

${ }^{3}$ Bundesinstitut für gesundheitlichen Verbraucherschutz und Veterinärmedizin,Berlin

${ }^{4}$ Bundesamt für Strahlenschutz, Berlin

\title{
Aktionsprogramm Umwelt und Gesundheit
}

\section{Zusammenfassung}

Das gemeinsame Aktionsprogramm „Umwelt und Gesundheit" des Ministeriums für Gesundheit und des Ministeriums für Umwelt, Naturschutz und Reaktorsicherheit löst die eingegangene Verpflichtung ein, die aus den europäischen Umweltkonferenzen resultiert. Das Programm dient dem Ziel, eine Handlungsbasis aufzustellen, auf welcher der Gesundheitszustand der Bürger und Bürgerinnen in Bereichen sichergestellt wird, die durch ungünstige Einflüsse von Umweltnoxen physikalischer Natur, mediengetragenen Umweltnoxen oder durch Aufnahme beim Umgang mitVerbraucherprodukten destäglichen Bedarfs oder durch die Nahrung gefährdet sein können. Das Programm wurde auf dem Hintergrund eines ausführlichen Dokumentationsbandes erstellt, der den aktuellen Sach- und Erkenntnisstand ausführlich darstellt und damit die vorgeschlagenen Ziele und Maßnahmen inhaltlich begründet. Das Programm enthält folgende Themen: umweltbezogene Gesundheitsbeobachtung und -berichterstattung, Informationsmanagement, Umgang mit Risiken, Umweltmedizin, Verbesserung der bestehenden Behördenstrukturen und internationaleZusammenarbeit. Das Programm enthält zudem verschiedene medien- und stoffbezogene Qualitätsziele zu folgenden Themen: Außenluft und Klima; Innenraumluft; Wasserressourcen, Boden, Lebensmittel, ionisierende Strahlung; Lärm sowie Stoffe und Zubereitungen.Das Programm soll eine Arbeitsgrundlage für die weitere Entwicklung des Politikfeldes Umwelt und Gesundheit bilden. Die weitere Ausgestaltung und Umsetzung des Programms wird in intensivem Diskurs mit allen Beteiligten und Betroffenen erfolgen.

\section{Schlüsselwörter}

Umwelt und Gesundheit · Aktionsprogramm . Umweltmedizin - Risikomanagement gesundheitsbezogene Umweltqualitätsziele
M men zum Schutz der Umwelt dienen im weitesten Sinne auch dem Schutz der Gesundheit. Aktivitäten in beiden Bereichen verfolgen daher häufig gemeinsame Ziele und erfordern folglich dann auch gemeinsame Strategien und Maßnahmen. Zur Förderung der Kooperation beider Politikbereiche und mit dem Ziel einer umfassenden Auseinandersetzung mit den gesundheitlichen Folgen von Umwelteinwirkungen haben das Bundesministerium für Umwelt, Naturschutz und Reaktorsicherheit und das Bundesministerium für Gesundheit gemeinsames Handeln beschlossen.

\section{„Jeder Mensch hat Anspruch auf eine Umwelt, die ein höchstmögliches Maß an Gesundheit und Wohlbefinden ermöglicht."}

Die Mitgliedsstaaten der europäischen Region der WHO erkannten bereits 1984 die Bedeutung des Zusammenwirkens der für die beiden Politikbereiche Verantwortlichen und definierten prioritäre Ziele für den umweltbezogenen $\mathrm{Ge}$ sundheitsschutz im Rahmen der Strategie „Gesundheit für alle”. Ausgehend von den WHO-Aktivitäten fand 1989 in Frankfurt/Main die von Deutschland aktiv unterstützte 1. Europäische Konferenz „Umwelt und Gesundheit” statt. Auf dieser Konferenz wurde die „Europäische Charta Umwelt und Gesundheit" verabschiedet, die u.a. den Grundsatz enthält, dass jeder Mensch Anspruch auf eine Umwelt hat, die ein höchstmögliches Maß an Gesundheit und Wohlbefinden ermöglicht. Anlässlich der 2. Europäischen Konferenz 1994 in Helsinki legte die WHO einen „Aktionsplan Um- welt und Gesundheit für Europa" (EHA$\mathrm{PE})$ vor. Die Umwelt- und Gesundheitsminister der teilnehmenden Staaten einigten sich darauf, diesen Plan auf nationaler Ebene durch „Nationale Aktionspläne Umwelt und Gesundheit” (NEHAPs) umzusetzen. Zur 3. Europäischen Konferenz „Umwelt und Gesundheit”, die 1999 in London stattfand, legten das Bundesministerium für Umwelt, Naturschutz und Reaktorsicherheit (BMU) und das Bundesministerium für $\mathrm{Ge}$ sundheit (BMG) als gemeinsamen nationalen Beitrag das „Aktionsprogramm Umwelt und Gesundheit" vor.

\section{Inhalt des Aktionsprogramms}

Das Aktionsprogramm will zur Vertiefung des Verständnisses über die $\mathrm{Zu}$ sammenhänge zwischen Umwelt und Gesundheit sowie zur Verbesserung der Information und Kommunikation $\mathrm{zu}$ gesundheitlichen Risiken beitragen und listet Aktivitäten auf, die diesem Ziel dienen. Zudem schlägt das Programm Maßnahmen zur Risikovermeidung oder Risikominderung für sechs prioritäre, durch Qualitätsziele charakterisierte Bereiche vor. Diese Bereiche sind: Außenluft und Klima, Innenraumluft, Wasserressourcen/Boden/Lebensmittel, Ionisierende Strahlung, Lärm sowie Stoffe und Zubereitungen.

$\mathrm{Zu}$ dem Aktionsprogramm gehört eine Dokumentation, die den aktuellen Sach- und Erkenntnisstand ausführlich darstellt und damit die vorgeschlagenen Ziele und Maßnahmen im Aktionsprogramm begründet (vgl. Abschnitt „Dokumentationsband zum Aktionsprogramm").

Dr. Bernd Seifert

Umweltbundesamt, Corresplatz 1, 14195 Berlin 
Bundesgesundheitsbl -

Gesundheitsforsch - Gesundheitsschutz

2000 • 43:323-327 @ Springer-Verlag 2000

B. Seifert · H. Schreiber - B. Bellach • U.Gundert-Remy $\cdot$ T.Jung

Action programme „environment and health"

\section{Abstract}

In fulfilling the commitment to the European Conferences on Environmental Health the Federal Ministry for Health and the Federal Ministry for the Environment, Nature Conservation and Nuclear Safety presented a joint "Action Programme on Environment and Health". The action programme is backed up by a comprehensive documentation presenting the current status and findings and providing justification for the proposed goals and measures. The programme covers the following subjects: environmental health monitoring and reporting, information management, risk perception and risk acceptance, environmental medicine, measures to improve co-operation among competent national authorities, international co-operation. The programme also conveys media- and substance-oriented quality objectives, in particular concerning outdoor air and climate; indoor air; water resources, soil, food; ionising radiation; noise; substances and preparations. The programme is aimed at supporting the policy development in the field of environment and health. The planning of detailed steps and the programme's implementation will be performed in close co-ordination and cooperation of all parties interested/involved and afflicted/affected.

\section{Keywords}

Environment and Health · Action Programme · Environmental Medicine · Risk Management $\cdot$ Health Related Environmental Quality Objectives

Leitthema: Umwelt und Gesundheit

Ziel des Aktionsprogramms ist es, den gesundheitlichen Umweltschutz und die Umweltmedizin im Rahmen einer Gesamtstrategie auf eine tragfähige, den aktuellen und zukünftigen Erfordernissen entsprechende Basis zu stellen. Das Programm soll insbesondere auch dazu beitragen, die Managementstrategien und die Strukturen zur Bearbeitung des Querschnittsbereichs Umwelt und Gesundheit zu verbessern. Das Programm bildet die Arbeitsgrundlage für die weitere Entwicklung des Politikfeldes Umwelt und Gesundheit. Das Programm ist in zwei Hauptteile gegliedert:

Deil I: Ziele und Querschnittsmaßnahmen

Deil II: Medien- und stoffbezogene Qualitätsziele

\section{Ziele und Querschnittsmaßnahmen}

Der erste Teil des Aktionsprogramms umfasst Strategien und Maßnahmen, die das Verständnis über die Zusammenhänge zwischen Umwelt und Gesundheit vertiefen und die Information und Kommunikation zu den entsprechenden gesundheitlichen Risiken verbessern sollen.

Verbesserung einer umweltbezogenen Gesundheitsbeobachtung und -berichterstattung

Ziel dieses Schwerpunktes ist es, die koordinierte Beobachtung von Umwelt und Gesundheit und der zwischen beiden Bereichen bestehenden Zusammenhänge sowie die Berichterstattung $\mathrm{zu}$ verbessern, um ein differenziertes Beobachtungs- und Berichterstattungs(„Surveillance”-) System für Umwelt und Gesundheit zu schaffen. Beispielhaft sei die begonnene Verknüpfung von Umwelt- und Gesundheits-Survey genannt.

\section{Verbesserung des \\ Informationsmanagements}

Im Rahmen dieses Schwerpunktes soll die Zusammenarbeit zwischen den beteiligten Bundesoberbehörden intensiviert und ein gemeinsames aktives Informationsmanagement entwickelt werden (vgl. Beitrag von U. Kaiser). Hierzu gehören die Fortführung und der Ausbau des Umweltmedizinischen Informationsforums (UmInfo), das von BMU/BMG gefördert wird, sowie des vom Robert Koch-Institut betreuten Umweltmedizinischen Informationssystems (UmIS).

\section{Umgang mit Risiken}

Als besonders wichtig werden Fragen im Zusammenhang mit Risikobetrachtungen angesehen. Hierzu richten die beiden Ministerien eine ad-hoc-Kommission „Neuordnung der Verfahren und Organisationsstrukturen zur Risikobewertung und Standardsetzung" ein, die vom BfS betreut wird (vgl. Beitrag von Jung und Jahraus). Die Kommission soll innerhalb von zwei Jahren Vorschläge zur Neuordnung der Beratungsgremien sowie für ein transparenteres Verfahren der Standardsetzung unter angemessener Beteiligung der gesellschaftlich relevanten Gruppen und zur Verbesserung der Zusammenarbeit zwischen den beteiligten Behörden erarbeiten.

\section{„Mit einer aktiven und früh- zeitig einsetzenden Risiko- kommunikation soll in der öffentlichkeit ein besseres Verständnis für umwelt- und gesundheitspolitische Entscheidungen erreicht werden."}

Besondere Aufmerksamkeit gilt der Entwicklung einer aktiven und frühzeitig einsetzenden Risikokommunikation, um in der Öffentlichkeit ein besseres Verständnis für die umwelt- und gesundheitspolitische Entscheidungsfindung zu erreichen.

\section{Umweltmedizin}

Dieser Schwerpunkt dient der Förderung von Informationsaustausch, Qualitätssicherung und Forschung in der Umweltmedizin, um die medizinische Betreuung von Personen mit umweltbezogenen Gesundheitsstörungen sicherzustellen (vgl. Beitrag von Eis) Defizite bestehen u.a. in der Qualitätssicherung und in der Evaluation der in der Umweltmedizin angewandten diagnosti- 
schen und therapeutischen Verfahren. Im Rahmen dieses Schwerpunktes soll insbesondere eine zentrale Erfassungsund Bewertungsstelle für umweltmedizinische Methoden aufgebaut werden.

\section{Förderung der Forschung zu Umwelt} und Gesundheit

Die Forschung auf dem Gebiet Umwelt und Gesundheit soll auf hohem Niveau erhalten und gefördert werden. Im Rahmen bestehender Forschungskonzepte und Förderprogramme sollen eine Reihe von Fragen besonders aufgegriffen werden. Beispielhaft seien genannt: umweltassoziierte Gesundheitsstörungen, Kindergesundheit und Umwelt sowie Evaluation von Monitoring-Systemen.

\section{Überprüfung der bestehenden Behördenstrukturen}

Ziel des Aktionsprogramms ist auch, die vorhandenen Behördenstrukturen im Bereich Umwelt und Gesundheit zu überprüfen und ggf. neu zu gestalten, um eine optimale Arbeitsteilung und eine flexible Bearbeitung aktueller Probleme zu erreichen.

\section{Internationale Zusammenarbeit}

Im Rahmen dieses Schwerpunktes soll dazu beigetragen werden, weltweit gesundheitsförderliche Umweltbedingungen zu erhalten oder zu erreichen. Hierzu soll insbesondere der wissenschaftliche Austausch auf internationaler Ebene und die Mitarbeit in internationalen Gremien verstärkt werden. Mit der Einrichtung des WHO-Koordinierungszentrums für Information und Kommunikation am Robert Koch-Institut ist dazu ein wichtiger Schritt getan worden (vgl. Beitrag von E. Tschishowa).

\section{Medien- und stoffbezogene Qualitätsziele}

Der zweite Teil des Aktionsprogramms befasst sich mit sechs prioritären Bereichen, für die Qualitäts- und Handlungsziele sowie verschiedene Teilziele und Maßnahmen genannt werden.

\section{Außenluft und Klima}

Zur Erreichung des Ziels einer flächendeckend gesundheitsverträglichen Luft- güte sollen die Konzentrationen von Luftverunreinigungen weiter herabgesetzt werden. Besonders genannt werden kanzerogene Luftschadstoffe, bodennahes Ozon, Feinstäube, Treibhausgase und Ozonschicht-schädigende Stoffe.

\section{Innenraumluft}

Die Innenraumluft wird durch Schadstoffe aus z.B. Bauprodukten, Einrichtungsgegenständen, Haushaltschemikalien, Bioziden und Tabakrauch belastet. Für das angestrebte Ziel einer gesundheitlich einwandfreien Luftqualität in Innenräumen werden neben eigenverantwortlichen Maßnahmen der Verbraucher auch staatliche Aktivitäten zur Minderung schädlicher Emissionen als notwendig angesehen (siehe auch Beitrag von A. Hahn).

\section{Wasserressourcen, Boden, Lebensmittel}

Verunreinigungen im Trinkwasser und in Lebensmitteln kommen heute im Allgemeinen in gesundheitlich unbedenklichen Mengen vor. Dennoch sind einige Problembereiche verblieben, die der weiteren Bearbeitung bedürfen, um das Ziel der flächendeckenden Erhaltung von Wasser und Boden in gesundheitlich unbedenklicher Qualität zu erreichen.

\section{Ionisierende Strahlung}

Zur Minimierung der Belastung des Menschen durch ionisierende Strahlung sind insbesondere die Radonemissionen in Innenräumen zu verringern. Radonbelastungen tragen zur Entstehung von Lungenkrebs bei. Auch die Strahlenbelastungen durch radiologische Verfahren in der Medizin sollten unter Abwägung des diagnostischen Nutzens für den Patienten gegen die Strahlenrisiken weiter reduziert werden.

\section{Lärm}

Die allgemeine Geräuschbelastung der Bevölkerung ist erheblich und erfordert insbesondere im Verkehrsbereich Maßnahmen, um das angestrebte Ziel - die Minderung des Lärms auf gesundheitsverträgliche Werte - zu erreichen.

\section{Stoffe und Zubereitungen}

Zum Schutz der menschlichen Gesundheit vor schädlichen Wirkungen durch Stoffe und Zubereitungen sollen insbesondere hormonartig wirkende Stoffe, Biozide und Arzneimittel betrachtet werden.

Auch die Ausführungen zu den medien- und stoffbezogenen Qualitätszielen enthalten eine Vielzahl von Vorschlägen für Maßnahmen zur Reduzierung der gesundheitlichen Belastung. Detailliertere Angaben sind im Dokumentationsband zum Aktionsprogramm zu finden (vgl. nächster Abschnitt).

\section{Dokumentationsband zum Aktionsprogramm}

Die im Aktionsprogramm Umwelt und Gesundheit enthaltenen Ziele und Maßnahmen basieren auf dem Sach- und Erkenntnisstand, der in einem Dokumentationsband zum Aktionsprogramm zusammengestellt ist. Der Band wurde von den fachlich zuständigen wissenschaftlichen Bundesoberbehörden - Umweltbundesamt (UBA), Bundesinstitut für gesundheitlichen Verbraucherschutz und Veterinärmedizin (BgVV), Robert Koch-Institut (RKI) und Bundesamt für Strahlenschutz (BfS) - unter Mitwirkung eines interdisziplinär zusammengesetzten, von den Ministerien BMU und BMG berufenen Beraterkreises zum Aktionsprogramm erarbeitet.

Nach einer Einführung, in der das politisch-gesellschaftliche Umfeld $\mathrm{zu}$ Umwelt und Gesundheit in Deutschland und die nationalen und internationalen Aktivitäten, die zur Erarbeitung des Aktionsprogramms führten, dargestellt werden, geht der Dokumentationsband in drei Hauptkapiteln auf verschiedene Aspekte schwerpunktmäßig ein:

D Darstellung des fachlichen Hintergrundes zu Umwelt und Gesundheit, D Diskussion ausgewählter gesundheitsrelevanter Umweltprobleme,

- Benennung von Strategien und Maßnahmenvorschlägen.

Im ersten Kapitel wird insbesondere untersucht, welchen Einfluss die anthropogen veränderte Umwelt auf die Gesundheit hat. Dabei werden verschiedene $\mathrm{Ge}$ sundheitsstörungen betrachtet, die häufig mit Einflüssen aus der Umwelt in $\mathrm{Zu}$ sammenhang gebracht werden: Atem- 
wegserkrankungen, Krebs, Allergien, Störungen der Fertilität und Reproduktionsfähigkeit des Menschen und HerzKreislaufstörungen. Ein gesonderter Abschnitt ist umweltassoziierten Gesundheitsstörungen im engeren Sinne gewidmet („Umweltsyndrome”): ,sick-building"-Syndrom (SBS), Multiple Chemical Sensitivity (MCS), Chronic Fatigue Syndrome (CFS), Fibromyalgie-Syndrom (FMS) und elektromagnetische Hypersensibilität.

Trotz intensiver Forschung und beachtlicher Verbesserung des Erkenntnisstandes in den letzten Jahren ist die Bewertung umweltbedingter Erkrankungen und die Ermittlung des Beitrags einzelner Umweltfaktoren noch immer problematisch. Dies gilt insbesondere für chronische Effekte und für Effekte, bei denen eine lange Latenzzeit zwischen Ursache und Wirkung besteht. Vor allem aus Gründen der Vorsorge ist es daher notwendig, durch weitere wissenschaftliche Arbeiten eine genauere Bewertung zu ermöglichen.

\section{„Irotz intensiver Forschung ist die Bewertung umwelt- bedingter Erkrankungen und einzelner Umweltfaktoren noch immer problematisch."}

Besondere Bedeutung im Themenfeld Umwelt und Gesundheit kommt der Umweltmedizin zu. Die derzeit verfügbaren diagnostischen Möglichkeiten und die methodisch bedingten Grenzen werden daher im Dokumentationsband dargestellt (vgl. Beitrag von D. Eis).

Zum besseren Verständnis der einzelnen Kapitel des Dokumentationsbandes werden im ersten Hauptteil des Bandes auch eine Reihe von fachlichen Grundlagen, so z.B. der Epidemiologie, der Toxikologie und der Risikobetrachtung im wissenschaftlichen und gesellschaftspolitischen Kontext, dargestellt.

Ausführlich wird auf die derzeit vorhandenen Datenquellen eingegangen, sei es auf dem Gesundheitssektor (z.B. Krebs- oder Fehlbildungsregister) oder auf dem Umweltsektor (z.B. der gesundheitsbezogenen Umweltbeobachtung oder Erhebungen zu Altlastenvorkommen). Hier zeigt sich auch die Bedeutung einer Verknüpfung dieser beiden Bereiche. Die Ausführungen zu den Datenquellen werden ergänzt durch ei- nen Exkurs über die verfügbaren Informations- und Kommunikationssysteme (vgl. Beitrag von U. Kaiser).

Das zweite Kapitel des Dokumentationsbandes stellt die gesundheitsrelevanten Umweltprobleme dar: Lärm, Außen- und Innenraumluft, Strahlung, Wasser (Grund-, Trink-, Bade- und Abwasser), Boden und Altlasten, Siedlungshygiene, Lebensmittel und Bedarfsgegenstände, ausgewählte Produkte (Bauprodukte, Haushaltschemikalien, Biozide und Pflanzenschutzmittel) sowie ausgewählte Umweltnoxen (Schwermetalle, persistente organische Verbindungen). Ebenfalls betrachtet werden Störfälle, d.h. Chemie-, Industrie- oder Transportunfälle, da diese oftmals eine große Zahl von Menschen betreffen und meist erhebliche Folgen für Gesundheit und Umwelt haben können.

Das Kapitel schließt mit einer Betrachtung der gesundheitsrelevanten Umweltprobleme, die mit globalen Umweltveränderungen verbunden sein können. Näher beleuchtet wird die gesundheitliche Bedeutung der Zerstörung der Ozonschicht, insbesondere die Wirkung einer Zunahme der bodennahen UV-Strahlung auf die Augen, Haut und das Immunsystem. Erörtert werden auch die Zusammenhänge zwischen globaler Klimaveränderung und menschlicher Gesundheit. Als Beispiele werden einerseits die Möglichkeit einer meteorologisch bedingten Zunahme der Konzentration von Pollen, Bakterien und Pilzsporen in der Luft und die daraus eventuell resultierende Erhöhung der Zahl allergischer Erkrankungen genannt und andererseits ein möglicherweise erhöhtes Infektionsrisiko bei Verbesserung der Lebensbedingungen für manche Krankheitserreger bei einer generellen Erwärmung diskutiert.

Jeder der Abschnitte über die einzelnen gesundheitsrelevanten Umweltprobleme schließt mit einer Reihe von Empfehlungen, die auch die Grundlage zu den im Aktionsprogramm selbst aufgeführten Qualitätszielen und Maßnahmen bilden.

Das dritte Kapitel des Dokumentationsbandes stellt Strategien und Maßnahmenvorschläge vor, die vor allem dazu gedacht sind, die Bearbeitung des gesamten Problembereiches „Umwelt und Gesundheit" in der Zukunft zu erleichtern und zu verbessern. Angesprochen werden Fragen der umweltbezogenen
Gesundheitsbeobachtung bzw. der gesundheitsbezogenen Umweltberichterstattung einschließlich eines Konzepts für ein Umwelt-Gesundheits-Surveillance-System (s.u.). Möglichkeiten zur Verbesserung und Harmonisierung von Risikobewertung und Standardsetzung sowie des Informationsmanagements und Wege zur Kommunikation von Risiken in der Öffentlichkeit und einer geeigneten Bürgerbeteiligung bilden einen weiteren Schwerpunkt des Kapitels (siehe auch Beitrag von T. Jung, H. Jahraus und W. Burkart). In einem gesonderten Abschnitt werden ferner die bereits im ersten Hauptkapitel des Bandes angesprochenen Fragen hinsichtlich der Stellung und Leistungsfähigkeit der Umweltmedizin vertieft. Ein abschließender Abschnitt ist der Offenlegung der Wissens- und Forschungsdefizite gewidmet und enthält zahlreiche Empfehlungen für Forschungsprojekte.

\section{Strukturen zur Umsetzung des Aktionsprogramms}

Die Verantwortung für die Durchführung und Umsetzung des Aktionsprogramms obliegt dem Bundesministerium für Umwelt, Naturschutz und Reaktorsicherheit (BMU) und dem Bundesministerium für Gesundheit (BMG). Die beiden Ministerien haben eine Steuerungsgruppe ins Leben gerufen, in der auch die beteiligten Bundesoberbehörden vertreten sind.

Aufgabe der Steuerungsgruppe ist die Abstimmung und Organisation der Umsetzung der Maßnahmen des Aktionsprogramms. Auf der Ebene der beteiligten Bundesoberbehörden UBA, BgVV, RKI und BfS besteht eine Koordinierungsgruppe mit einer Geschäftsstelle beim UBA. Der Vorsitz der Koordinierungsgruppe wechselt im jährlichen Turnus zwischen den Behörden und liegt derzeit beim Umweltbundesamt. Aufgabe der Koordinierungsgruppe ist die wissenschaftliche Unterstützung bei der Umsetzung des Aktionsprogramms.

Zur politischen Beratung bei der Umsetzung und Weiterentwicklung der Zielsetzungen des Aktionsprogramms haben die Ministerien einen Beraterkreis mit externen Experten unter Beteiligung von Nicht-Regierungsorganisationen berufen. Zur fachlichen Begleitung von Einzelprojekten können bei Bedarf Fachgespräche durchgeführt und 
Projektgruppen gebildet werden, auch unter Hinzuziehung weiterer nationaler und internationaler Experten.

Teile der im Aktionsprogramm definierten Aufgaben fallen in die Zuständigkeit der Bundesländer oder können nur gemeinsam mit den Ländern bearbeitet werden. Die Zusammenarbeit mit den Bundesländern erfolgt über die Länderarbeitsgemeinschaft umweltbezogener Gesundheitsschutz (LAUG), in der sich eine Projektgruppe zur Umsetzung des Aktionsprogramms auf Länderebene gebildet hat. Steuerungs- und Koordinierungsgruppe sowie LAUGProjektgruppe werden bei der Ausführung des Aktionsprogramms eng zusammenarbeiten.

\section{Ad hoc-Kommission „Neu- ordnung der Verfahren und Organisationsstrukturen zur Risikobewertung und Standardsetzung"}

Ein zentraler Themenschwerpunkt des Aktionsprogramms Umwelt und Gesundheit ist der Umgang mit umweltbedingten Gesundheitsrisiken. In der Gesellschaft, der Politik, aber auch in der Wissenschaft wird über Umweltrisiken, ihre Auswirkungen auf die Gesundheit und ihre Akzeptierbarkeit kontrovers diskutiert. Sich widersprechende Meldungen in der Presse über neue Gefahrstoffe und Risiken können auf tatsächlich bestehende Wissenslücken hindeuten. Es fällt aber schwer, den Ursprung vermeintlich widersprüchlicher Sachstände, die oft zusammen in der selben Diskussion vorgetragen werden, zu erkennen. Informationen, Meinungen und Überzeugungen stehen oft nebeneinander und sind nur schwer zu unterscheiden. Eine kaum zu überblickende Fülle tatsächlicher und behaupteter Risikofaktoren erschwert eine rationale Prioritätensetzung in der Umweltpolitik und damit die Auseinandersetzung mit denjenigen Risiken, die im Sinne eines umfassenden und nachhaltigen Umweltund Gesundheitsschutzes eine intensive Befassung tatsächlich erfordern.

\section{„Über Umweltrisiken, ihre Aus- wirkungen auf die Gesundheit und ihre Akzeptierbarkeit wird in der Gesellschaft kontrovers diskutiert."}

Eine fundierte Bewertung von Risiken im Umwelt- und Gesundheitsschutz und die dazu gehörigen Verfahren der Standardsetzung sind zentrale Steuerungsinstrumente der Umwelt- und Gesundheitspolitik. Während Risikoabschätzung primär ein wissenschaftlicher Prozess ist, werden Risikobewertung und Standardsetzung in erheblichem Umfang von politisch-gesellschaftlichen Aspekten mitbestimmt. Risikobewertung aber auch Standardsetzung wurden bisher im Wesentlichen als eine alleinige Aufgabe für natur- und technikwissenschaftliche Spezialisten und Regulatoren gesehen. Betroffene Dritte waren bisher nicht eingebunden oder wurden höchstens als passive Adressaten der Risikokommunikation behandelt.

Um der politisch-gesellschaftlichen Dimension im gesundheitlichen Umweltschutz Rechnung zu tragen, müssen die Verfahren der Risikobewertung und besonders der Standardsetzung transparenter gestaltet werden. Dazu müssen relevante Gruppen aus Wissenschaft wie Gesellschaft auf einer möglichst breiten Basis einbezogen werden. Für Verfahren der Risikobewertung und Standardsetzung kann nur über eine solche Beteiligung die politische Legitimation in einer demokratischen Gesellschaft erreicht werden.

\section{„Die Verfahren der Risiko- bewertung und besonders der Standardsetzung müssen transparenter gestaltet werden."}

Eine hochrangig besetzte Kommission aus externen Experten und Fachvertretern der mit Risikoabschätzung und -bewertung sowie der Standardsetzung befassten wissenschaftlichen Behörden des Bundes soll Vorschläge entwickeln, wie die Verfahren der Risikobewertung und Standardsetzung im gesundheitlichen Umweltschutz harmonisiert und transparenter gestaltet werden können. Eine angemessene Beteiligung gesellschaftlich relevanter Gruppen soll bei zukünftigen Verfahren sichergestellt werden. Transparenz und Vergleichbarkeit von Risiken erfordern, dass Risikobewertungen unter Anwendung möglichst einheitlicher Qualitätskriterien in möglichst allen Regelungsbereichen des
Umwelt- und Gesundheitsschutzes erfolgen. Unter Berücksichtigung einer kritischen Analyse des gegenwärtigen Standes von Risikobewertung und Standardsetzung sollen Vorschläge zur Weiterentwicklung der Verfahrensweisen sowie der Beratungs- und Entscheidungsstrukturen bei der Ableitung von Umwelt- und Gesundheitsstandards erarbeitet werden.

Um bereits bei der Besetzung der Kommission einerseits dem Beteiligungsanspruch gerecht $\mathrm{zu}$ werden und andererseits die Hochrangigkeit der Kommissionsmitglieder zu gewährleisten, wurden die Dachverbände von im gesundheitlichen Umweltschutz tätigen Nicht-Regierungsorganisationen um Besetzungsvorschläge gebeten. Alle $\mathrm{zu}$ berufenden Mitglieder müssen über besondere Kenntnisse und Erfahrungen in der Risikobewertung und Standardsetzung auf naturwissenschaftlicher, gesellschaftswissenschaftlicher oder rechtswissenschaftlicher Ebene verfügen. In der Kommission sollen insbesondere die Querschnittsarbeitsgebiete Toxikologie, Strahlenbiologie, Umweltepidemiologie, Umweltmedizin, Umweltrecht, Umweltökonomie, Risikokommunikation und Umweltethik durch Expertinnen und Experten vertreten sein. Aus Gründen der Arbeitsfähigkeit wird die Kommission nicht mehr als 16 Mitglieder haben, davon nicht mehr als fünf aus mit Risikobewertung und Standardsetzung befassten wissenschaftlichen Behörden des Bundes. Die Kommission soll ihre Arbeit im Frühsommer 2000 aufnehmen und ihren Bericht dann innerhalb von zwei Jahren vorlegen. Die Bundesministerin für Gesundheit und der Bundesminister für Umwelt, Naturschutz und Reaktorsicherheit werden anhand der Vorschläge 16 Expertinnen und Experten als Mitglieder der ad hoc-Kommission zur Neuordnung der Verfahren und Strukturen zur Risikobewertung und Standardsetzung im gesundheitlichen Umweltschutz der Bundesrepublik Deutschland gemeinsam berufen. 